\title{
Optomechanical forces and electrostriction in laser optical materials
}

\author{
David L. Andrews and Richard G. Crisp \\ Nanostructures and Photomolecular Systems, School of Chemical Sciences, \\ University of East Anglia, Norwich NR4 7TJ, U.K.
}

\begin{abstract}
With optical tweezer methods now firmly established and the nature of optical forces on individual particles well understood, one of the separate but related issues that has only recently come to the fore concerns the effects of intense optical radiation on inter-particle forces. It has already been established that such forces, which are not dependent on optical field gradients, can effect a weak binding between particles leading in some cases to optical clustering and in others to pattern formation. In this presentation it is shown by quantum electrodynamical analysis that a variety of other optomechanical effects can be produced in materials or systems subjected to the throughput of intense, non-resonant laser radiation. In particular, an optical electrostriction phenomenon is identified and shown to be widely operative in laser optical materials. Although a classical electrodynamical interpretation (in terms of interactions between induced dipoles) comfortably predicts the sign of the resulting mechanical force, it is shown that such a picture has significant limitations in addressing this fundamentally photonic phenomenon. The key parameters that determine the size and character of optical electrostriction are delineated and its significance is quantitatively assessed. The experimental challenges involved in characterizing such phenomena are also given a detailed appraisal.
\end{abstract}

Keywords: Optomechanical forces, electrostriction, laser optics, Casimir-Polder interaction, quantum electrodynamics

\section{INTRODUCTION}

The use of laser light to manipulate and guide small particles is an increasingly prominent research tool, finding applications in diverse laboratories. A variety of optomechanical mechanisms operate, across a spectrum of techniques that ranges from optical tweezers to laser cooling and trapping, though all are based on forces that operate directly on individual particles. Recently a wave of excitement has been created by a discovery of an entirely different class of optically induced forces, which operate between particles, over nanoscale dimensions. ${ }^{1-10}$ Such forces offer a number of highly distinctive features which can be exploited for the controlled optical manipulation of matter. Through such interactions, new opportunities for creating optically ordered matter have already been demonstrated both theoretically and experimentally, leading to the introduction of terms such as 'optical binding' and 'optical matter' in the recent literature. ${ }^{11-13}$ Though slightly misleading, these are terms that rightly draw attention to new, intriguing and distinctive phenomena.

The existence of optically induced inter-particle forces was in fact first demonstrated in $1980^{14}$ through the application of quantum electrodynamics (QED), a framework of theory exceptionally well suited to the rigorous characterization of such intricate photonic interactions. The following years saw occasional studies by a number of research groups, based on different methods, ${ }^{15-20}$ but fresh interest arose on recognition that the levels of intensity originally considered necessary (typically megawatts per square centimeter) are now routinely available. There is now good reason to expect significant effects to be experimentally demonstrable at much lower intensity levels. Last year the UEA quantum electrodynamics group published the first fully comprehensive theory of optically induced interparticle force, based on QED. ${ }^{21}$ Specific calculations were also performed for carbon nanotubes, ${ }^{22}$ and the practicality of measuring significant laser-induced forces and torques was proven. It was also shown that the use of wavefrontstructured light, such as Laguerre-Gaussian beams, offers further scope to tailor the pattern of such forces and torques; it can, for example, provide a means of achieving particle ring formations. ${ }^{23}$

In work currently in press, ${ }^{24}$ we have shown how the mechanism for optical binding owes its origin to a modification of Casimir-Polder forces. One of the possible manifestations that we have recently begun to consider is the effect of intense throughput optical radiation on optically transparent solids, where it is conceivable that effects extending to the 
microscale might arise from the combined influence of light on the nearest-neighbor interactions between constituent particles of matter (atoms or molecules). It is the further exploration and quantification of this solid-state effect that we report below.

\section{QUANTUM ELECTRODYNAMICAL DEVELOPMENT}

To develop the following theory we consider a regular solid comprising close-packed, electrically neutral molecules. It is assumed that the material is essentially transparent to the radiation that will be used to induce mechanical response. In such a system the intrinsic lattice structure provides for a counterbalance of the intermolecular electronic repulsion and London attraction forces between the constituent particles, in the absence of throughput radiation. As will be shown, the additional, optically-induced forces and resulting effects that can be calculated for such a system prove readily measurable. Larger and more easily measured effects might be anticipated in other kinds of solid, especially any with metallic constituents, but to address such electronically more responsive (and absorptive) systems will require a further, major development of theory - which will represent the next stage of theoretical development. Here we analyze and derive results for the simplest case, to establish the principles and to encourage experimental investigations.

We first outline the means of deriving, through quantum electrodynamics, an expression for the energy shift associated with the engagement of off-resonant radiation with the coupling between a neighboring pair of molecules $A$ and $B$. Representing the system in quantum electrodynamical terms in the Coulomb gauge ensures that the coupling fields are duly retarded and satisfy causality. In multipolar form the system Hamiltonian may be represented thus;

$$
H=\sum_{\xi=\mathrm{A}, \mathrm{B}} H_{\mathrm{mol}}^{\xi}+\sum_{\xi=\mathrm{A}, \mathrm{B}} H_{\mathrm{int}}^{\xi}+H_{\mathrm{rad}}
$$

Here $H_{\mathrm{mol}}^{\xi}$ is the field-free multipolar Hamiltonian for molecule $\xi$, operator $H_{\text {int }}^{\xi}$ represents the interaction of $\xi$ with the radiation field, and $H_{\text {rad }}$ is the radiation Hamiltonian. The tripartite simplicity of equation (1) specifically results from adoption of the multipolar form of light-matter interaction, based on a well-known canonical transformation from the minimal-coupling interaction. This procedure results in a precise cancellation from the system Hamiltonian of all Coulombic terms, save those intrinsic to the internal structure of the Hamiltonian operators for the component molecules. In the electric dipole approximation, we have;

$$
H_{\mathrm{int}}^{\xi}=-\varepsilon_{0}^{-1} \sum_{\xi} \mu(\xi) \cdot \mathbf{d}^{\perp}\left(\mathbf{R}_{\xi}\right)
$$

Here $\mu(\xi)$ and $\mathbf{R}_{\xi}$ denote the electric-dipole operator and the position vector of molecule $\xi$, respectively; $\mathbf{d}^{\perp}\left(\mathbf{R}_{\xi}\right)$ represents the transverse electric displacement field operator at that location. As shown elsewhere, ${ }^{21}$ the leading term in the result for the optically induced energy-shift, $\Delta E$, emerges from fourth-order perturbation theory;

$$
\Delta E=\operatorname{Re}\left\{\sum_{t, s, r} \frac{\left\langle i\left|H_{\mathrm{int}}\right| t\right\rangle\left\langle t\left|H_{\mathrm{int}}\right| s\right\rangle\left\langle s\left|H_{\mathrm{int}}\right| r\right\rangle\left\langle r\left|H_{\mathrm{int}}\right| i\right\rangle}{\left(E_{i}-E_{t}\right)\left(E_{i}-E_{s}\right)\left(E_{i}-E_{r}\right)}\right\} .
$$

Here all states are those of the system, i.e. the two molecules plus the radiation field; $i$ is the unperturbed system state in which both molecules are in their electronic ground state; $r, s$ and $t$ are virtual states, and $E_{n}$ is the energy of state $n$. The latter signifies one of the basis states for the perturbative development, expressible in the form;

$$
|n\rangle=\left|\operatorname{mol}_{n}\right\rangle\left|\operatorname{rad}_{n}\right\rangle \equiv\left|\operatorname{mol}_{n} ; \operatorname{rad}_{n}\right\rangle
$$

the product defining states of the molecular pair and the radiation, respectively. In equation (3), each operation of the interaction operator on the state to its right effects the annihilation or creation of a photon; the laser-induced interaction 


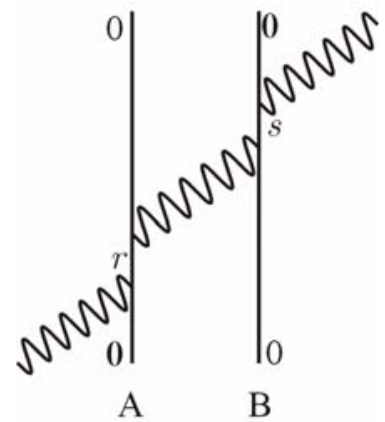

Figure 1: One of forty-eight time-ordered diagrams, generated by equation (3), together representing the complete set of quantum amplitudes for optically-induced coupling between a pair of particles $A$ and $B$. Time progresses upwards; solid vertical lines represent world-lines for each of the two particles, wavy lines denote electromagnetic quanta. Here a real (laser) photon impinges from the left and an identical mode photon propagates out on the right; the wavy line connecting A and B signifies a short-lived virtual photon. The ground electronic states of each particle are denoted by 0 , and $r, s$ are virtual states.

involves the annihilation of a throughput photon at one molecule and the stimulated emission of an equivalent 'real' photon at the other; this is mediated by intermolecular energy transfer through a virtual photon that is created at one site and annihilated at the other. The molecules and the throughput radiation suffer no overall change in state; the term 'real' applied to photons of the input denotes quanta of electromagnetic radiation with a propagation time that is long compared to the optical cycle. In performing energy shift calculations based on (3), detailed representations of all contributory terms are provided by a set of 48 time-ordered diagrams, one of which is exhibited in figure 1 . The explicit result for $\Delta E$ is concisely expressible as follows, defining $\mathbf{R} \equiv \mathbf{R}_{B}-\mathbf{R}_{A}$ as the inter-particle displacement vector, and using the implied summation convention for repeated Cartesian tensor indices;

$$
\Delta E(k, \mathbf{R})=\left(\frac{I}{\varepsilon_{0} c}\right) \operatorname{Re}\left\{e_{i}^{(\lambda)} \bar{e}_{l}^{(\lambda)} \alpha_{i j}^{\mathrm{A}}(k) V_{j k}^{ \pm}(k, \mathbf{R}) \alpha_{k l}^{\mathrm{B}}(k) \exp (\mathrm{ik} \cdot \mathbf{R})\right\} .
$$

Here $\mathbf{k}$ and $\hbar c k$ denote the wave-vector and photon energy, respectively, of the input of irradiance $I$; $\mathbf{e}^{(\lambda)}$ is the corresponding polarization unit vector (considered complex to allow for circular or elliptical as well as plane polarization - an overbar denoting complex conjugate). Also $V_{j k}^{ \pm}$signifies the fully retarded resonance electric dipole electric dipole interaction tensor, given by;

$$
V_{j k}^{ \pm}(k, \mathbf{R})=\frac{e^{\mp i k R}}{4 \pi \varepsilon_{0} R^{3}}\left\{(1 \pm \mathrm{i} k R)\left(\delta_{j k}-3 \hat{R}_{j} \hat{R}_{k}\right)-(k R)^{2}\left(\delta_{j k}-\hat{R}_{j} \hat{R}_{k}\right)\right\} .
$$

(The equal validity of either sign in the \pm is demonstrated in ref. 25). Before proceeding with the detail it is interesting to identify and briefly interpret a classical representation of the optically induced pair force (a matter to which we return in Section 4). To this end, we note that equation (5) can be cast in terms of induced electrical polarizations $P$ as follows;

$$
\Delta E(R)=\frac{1}{2} \operatorname{Re}\left\{V_{j k}^{ \pm}\left(n_{k} k, \mathbf{R}\right) P_{j}(k) \bar{P}_{k}(k) \exp (i \mathbf{k} . \mathbf{R})\right\},
$$

noting the classical correspondence $n \hbar c k / \varepsilon_{0} V \rightarrow E^{2} / 2$, where $E$ is the electric field of the radiation. For neighboring molecules, the short-range limit of the exponential is appropriate and we have:

$$
\Delta E(R)=\sigma_{j k}(k) P_{j}(k) \bar{P}_{k}(k) \equiv \frac{1}{2} \operatorname{Re}\left\{V_{j k}^{ \pm}\left(n_{k} k, \mathbf{R}\right) P_{j}(k) \bar{P}_{k}(k)\right\},
$$

which casts the coupling tensor $\sigma_{j k}$ as an effective local stress tensor. Taking the second derivative with respect to vector components of $\mathbf{R}$ (and for conciseness suppressing the $k$-dependence) the ensuing tensor $\tilde{\mathbf{x}}$ signifies the microscopic response; 


$$
\tilde{x}_{i l} \equiv \frac{\partial^{2} \Delta E}{\partial R_{i} \partial R_{l}}=X_{i j k l} P_{j} P_{k} \equiv \frac{\partial^{2}}{\partial R_{i} \partial R_{l}} \sigma_{j k} P_{j} \bar{P}_{k}
$$

In a solid with perfect elasticity, the second-order strain tensor would be directly proportional to $\tilde{\mathbf{x}}$; the above equation thus designates the fourth rank tensor $X_{i j k l}$ as an effective electrostrictive coefficient. Although the term 'electrostrictive' is usually employed in connection with static electric fields, ${ }^{26}$ we adopt the term 'optically electrostrictive' to signify the effect to be detailed below; in contrast to 'piezoelectric', the former term also signifies a quadratic dependence on the induced polarization - and a corresponding independence of the sign.

\section{RESULTS}

To apply the theory we consider a regular solid comprising close-packed, electrically neutral quasi-linear molecules. Focusing on one neighboring pair of molecules in detail, we consider specifically a pair of parallel, cylindrically symmetric molecules with a mutual separation vector $\mathbf{R}$ orthogonal to their 'long' molecular axes. Identifying $\mathbf{R}$ with the Z-axis and the molecular axis with the $\mathrm{X}$-direction, and assuming the system is irradiated with plane-polarized light, we define the polarization vector of throughput radiation in cylindrical coordinates as $\mathbf{e}=\sin \phi \cos \theta \hat{\mathbf{i}}+\sin \phi \sin \theta \hat{\mathbf{j}}+$ $\cos \phi \hat{\mathbf{k}}$, where $\phi$ and $\theta$ are the angles made by $\mathbf{e}$ with $\mathbf{R}$ and the molecular axis, respectively. From (5), the laserinduced energy shift experienced by this pair is;

$$
\begin{gathered}
\Delta E(R)=\frac{I}{\varepsilon_{0} C} \operatorname{Re}\left\{\sin ^{2} \phi \cos ^{2} \theta \alpha_{X X}^{A} V_{X X} \alpha_{X X}^{B}+\sin ^{2} \phi \sin ^{2} \theta \alpha_{Y Y}^{A} V_{Y Y} \alpha_{Y Y}^{B}\right. \\
\left.+\cos ^{2} \phi \alpha_{Z Z}^{A} V_{Z Z} \alpha_{Z Z}^{B}\right\} \cos (\mathbf{k} \cdot \mathbf{R})
\end{gathered}
$$

On differentiating (10) with respect to distance, using equation (6), and after taking the leading terms in the Taylor series expansions of $\cos k R$, sin $k R$ and $\cos \mathbf{k} . \mathbf{R}$ (effecting a correction to the result given in ref. 21) we obtain the following expression for the force induced between the particles, $\mathbf{F}_{\text {ind }}=-\partial \Delta E_{\text {ind }} / \partial \mathbf{R}$;

$$
\mathbf{F}_{\text {ind }}(R)=\frac{3 I}{4 \pi \varepsilon_{0}^{2} c R^{4}}\left[\alpha_{\perp}^{A} \alpha_{\perp}^{B}\left(\sin ^{2} \phi\left(2+\sin ^{2} \theta\right)-2\right)+\alpha_{\|}^{A} \alpha_{\|}^{B} \sin ^{2} \phi \cos ^{2} \theta\right],
$$

where $\alpha_{\perp}=\alpha_{Y Y}=\alpha_{Z Z}, \alpha_{\|}=\alpha_{X X}$. This is effectively the only mechanically operative pair force, since other interparticle forces in the solid are balanced at equilibrium. It is instructive to consider special geometric cases.

\section{Parallel pair}

The following results emerge for cases in which the polarization vector of the incident light is: (i) parallel to the molecular axis; (ii) parallel to the separation vector, (iii) orthogonal to both;

$$
\mathbf{F}_{\text {ind }}(R)=\left\{\begin{array}{rr}
\frac{3 I\left(\alpha_{\|}^{A} \alpha_{\|}^{B}\right)}{4 \pi \varepsilon_{0}^{2} c R^{4}}, & \text { (i) }(\phi=\pi / 2, \theta=0) \\
-\frac{3 I\left(\alpha_{\perp}^{A} \alpha_{\perp}^{B}\right)}{2 \pi \varepsilon_{0}^{2} c R^{4}}, & \text { (ii) }(\phi=0, \theta=\pi / 2) \\
\frac{3 I\left(\alpha_{\perp}^{A} \alpha_{\perp}^{B}\right)}{4 \pi \varepsilon_{0}^{2} c R^{4}}, & \text { (iii) }(\phi=0, \theta=0)
\end{array}\right.
$$


The positive sign for results (i) and (iii) signifies a repulsion tending to increase the pair separation, leading to expansion; in case (ii) the attractive force will generate a contraction. The strengths of the forces depends on the magnitudes of $\alpha_{\|}^{\xi}$ and $\alpha_{\perp}^{\xi}$, and for electronically prolate molecules, $\left(\alpha_{\|}^{\xi}>\alpha_{\perp}^{\xi}\right)$, the repulsion (i) is the largest force.

\section{End-to-end pair}

The above results account for only two dimensions of molecular packing in these anisotropic solids; it is also necessary to consider the forces on a pair of adjacent particles placed end-to-end. Again the results differ according to whether the polarization vector of the incident radiation is: $(v)$ parallel to, or $(v i)$ orthogonal to the intermolecular separation vector. Explicitly, the ensuing forces are as follows;

$$
\mathbf{F}_{\text {ind }}^{0}(R)= \begin{cases}-\frac{3 I\left(\alpha_{\|}^{A} \alpha_{\|}^{B}\right)}{2 \pi \varepsilon_{0}^{2} c R^{4}}, & (v)(\mathbf{e} \| \mathbf{R}) \\ \frac{3 I\left(\alpha_{\perp}^{A} \alpha_{\perp}^{B}\right)}{4 \pi \varepsilon_{0}^{2} c R^{4}}, & (v i)(\mathbf{e} \perp \mathbf{R})\end{cases}
$$

Thus, irradiating such a solid with plane-polarized light effects a contraction in the direction of the solid parallel to the polarization of the laser beam, and an expansion in the other two orthogonal dimensions, both linearly dependent on the irradiance of the laser.

In summary, a compression parallel to $\mathbf{e}$ together with an expansion in directions perpendicular to $\mathbf{e}$ produces a solid deformation characterized by an overall increase in volume that scales linearly with the irradiance $I$. This in turn signifies a change in the local density - and hence in the local refractive index - that can also be expected to scale linearly with $I$. Optical electrostriction thus leads to an optomechanically induced intensity-dependence in the refractive index.

\section{LIMITATIONS OF THE CLASSICAL INTERPRETATION}

The form of results exhibited in equations (12), (13) is superficially consistent with, and suggestive of, a classical electrodynamic interpretation. The oscillating electric field of the electromagnetic radiation induces exactly in-phase (and, if the molecules are similarly aligned, parallel) oscillating dipoles in both molecules. When $\mathbf{e}$ and $\mathbf{R}$ are orthogonal, these dipoles line up 'head-to-head' - accounting for the repulsive force exhibited - whereas when they are parallel, a 'head-to-tail' configuration produces a force that is attractive.

However useful such an interpretation is as a guide, it must not be taken too literally. The problem with such an interpretation is principally that it is either suggestive of an instantaneous coupling, which is of course at odds with the demands of causality and special relativity, or else it overplays a certain type of temporal sequence at the expense of others, such as that illustrated by the time-ordering in figure 2. Though completely counter-intuitive, the detailed QED theory nonetheless shows such contributions to the coupling to be just as important in the near-zone as fig. 1, for

Figure 2: Another member of the set of forty-eight timeordered diagrams, generated by equation (3) - compare figure 1. Here the first event in time order is emission of a virtual photon by particle $B$, through which $B$ itself enters a virtual state $s$; this is followed by (stimulated) emission of a real photon as the same particle re-enters its electronic ground state. On reaching particle $A$, the virtual photon effects transition of the former to a virtual state $r$; A's return to the electronic ground state is effected on absorption of a real photon from the throughput beam - which concludes the sequence.

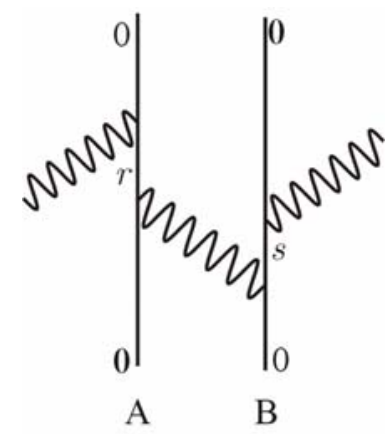


example. [The latter assertion has been explicitly proven for the case of resonance energy transfer (RET) - see ref 27 and the $\mathrm{V}$ tensor that appears in that connection is of precisely the same form as arises here.] The apparent paradox is resolved on recall of the time-energy uncertainty principle, and the ultrafast timescale associated with virtual photon propagation over nanoscale distances.

\section{DISCUSSION}

Detection of the process of optical electrostriction is fraught with experimental difficulty, and this is undoubtedly why it has not been characterized before. Such difficulties should not prove insurmountable, but they need to be taken into account in planning practicable experiments. Because the effect of interest leads to optical changes, there is an obvious temptation to seek an optical proof. However it is clear that the optical electrostrictive effect, in solids, will usually be dominated by much more widely known electronic mechanisms such as the optical Kerr ffect $^{28}-$ and, in absorbing regions, other photothermal effects due to spatially inhomogeneous heating. Intriguingly, electrostriction was indeed considered in connection with an intensity-dependent refractive index in the early days of laser physics, ${ }^{29}$ but it subsequently emerged that other mechanisms play a more direct and significant role.

The application of quantum electrodynamics has produced a clear physical picture of the fundamental interactions that can result in optical electrostriction, generating results that are readily quantified. Despite its relative unfamiliarity, it appears the process may operate to produce a significant levels of mechanical deformation in optically transparent solid materials subjected to intense laser light - hence the relevance to laser optical materials. Needless to say, such deformation would lead to changed optical characteristics; nonetheless the best prospects for observation are likely to be directly mechanical, most probably using atomic force microscopy methods.

Although we have focused on the intrinsic interest of the subject, it is worth considering the applicability of this effect to optomechanical devices. In the context of dramatically accelerating developments in the field of nanoelectromechanical systems, any mechanism that can reproducibly deliver a reversible and ultrafast mechanical response, actuated by light, appears to be of considerable merit. It is hoped the mechanism described herein will provide many opportunities and challenges in this burgeoning field.

\section{ACKNOWLEDGEMENT}

We are pleased to acknowledge funding from the Engineering and Physical Sciences Research Council.

\section{REFERENCES}

1. D. O'Dell, S. Giovanazzi, G. Kurizki and V. M. Akulin, "Bose-Einstein condensates with 1/r interatomic attraction: Electromagnetically induced "gravity"'", Phys. Rev. Lett., 84, pp. 5687-5690, 2000.

2. S. Giovanazzi, D. O'Dell and G. Kurizki, "One-dimensional compression of Bose-Einstein condensates by laserinduced dipole-dipole interactions", J. Phys. B: At. Mol. Opt. Phys., 34, pp. 4757, 2001.

3. S. Giovanazzi, D. O'Dell and G. Kurizki, "Self-binding transition in Bose condensates with laser-induced "gravitation"'", Phys. Rev. A, 6303, 2001.

4. S. Giovanazzi, D. O'Dell and G. Kurizki, "Density modulations of Bose-Einstein condensates via laser-induced interactions", Phys. Rev. Lett., 88, 2002.

5. I. E. Mazets, D. H. J. O'Dell, G. Kurizki, N. Davidson and W. P. Schleich, "Depletion of a Bose-Einstein condensate by laser-induced dipole-dipole interactions", J. Phys. B: At. Mol. Opt., 37, pp. S155-S164, 2004.

6. M. Nieto-Vesperinas, P. C. Chaumet and A. Rahmani, "Near-field photonic forces (vol 362, pg 719, 2004)", Philos. Trans. R. Soc. Lond. Ser. A-Math. Phys. Eng. Sci., 362, pp. 2889-2890, 2004.

7. A. Salam, "A general formula for the rate of resonant transfer of energy between two electric multipole moments of arbitrary order using molecular quantum electrodynamics", J. Chem. Phys., 122, 044112, 2005. 
8. A. Salam, "Resonant transfer of excitation between two molecules using Maxwell fields", J. Chem. Phys., 122, $044113,2005$.

9. J.-M. Fournier, J. Rohner, P. Jacquot, R. Johann, S. Mias and R.-P. Salathé, "Assembling mesoscopic particles by various optical schemes", Proc. SPIE, 5930, 59300Y, 2005.

10. A. Labeyrie, M. Guillon and J. M. Fournier, "Optics of laser trapped mirrors for large telescopes and hypertelescopes in space", Proc. SPIE, 5899, 58990V, 2005.

11. P. C. Chaumet and M. Nieto-Vesperinas, "Optical binding of particles with or without the presence of a flat dielectric surface", Phys. Rev. B, 6403, 035422, 2001.

12. S. K. Mohanty, J. T. Andrews and P. K. Gupta, "Optical binding between dielectric particles", Opt. Express, 12, pp. 2746-2753, 2004.

13. J.-M. R. Fournier, G. Boer, G. Delacretaz, P. M. Jacquot, J. Rohner and R. P. Salathé, "Building optical matter with binding and trapping forces", Proc. SPIE, 5514, pp. 309-317, 2004.

14. T. Thirunamachandran, "Intermolecular Interactions in the Presence of an Intense Radiation-Field", Mol. Phys., 40, pp. 393-399, 1980.

15. D. L. Andrews and M. J. Harlow, "Phased and Boltzmann-Weighted Rotational Averages", Phys. Rev. A, 29, pp. 2796-2806, 1984.

16. M. M. Burns, J. M. Fournier and J. A. Golovchenko, "Optical Binding", Phys. Rev. Lett., 63, pp. 1233-1236, 1989.

17. P. W. Milonni and M. L. Shih, "Source Theory of the Casimir Force", Phys. Rev. A, 45, pp. 4241-4253, 1992.

18. F. Depasse and J. M. Vigoureux, "Optical Binding Force between 2 Rayleigh Particles", J. Phys. D-Appl. Phys., 27, pp. 914-919, 1994.

19. J.-M. R. Fournier, M. M. Burns and J. A. Golovchenko, "Writing diffractive structures by optical trapping", Proc. SPIE, 2406, pp. 101-111, 1995.

20. P. W. Milonni and A. Smith, "van der Waals dispersion forces in electromagnetic fields", Phys. Rev. A, 53, pp. 3484-3489, 1996.

21. D. S. Bradshaw and D. L. Andrews, "Optically induced forces and torques: Interactions between nanoparticles in a laser beam", Phys. Rev. A, 72, 033816, 2005.

22. D. L. Andrews and D. S. Bradshaw, "Laser-induced forces between carbon nanotubes", Opt. Lett., 30, pp. 783785, 2005

23. D. S. Bradshaw and D. L. Andrews, "Interactions between spherical nanoparticles optically trapped in LaguerreGaussian modes", Opt. Lett., 30, pp. 3039-3041, 2005.

24. D. L. Andrews, R. G. Crisp and D. S. Bradshaw, "Optically induced inter-particle forces. From the bonding of dimers to optical electrostriction in molecular solids", J. Phys. B: At. Mol. Opt., (in press).

25. G. J. Daniels, R. D. Jenkins, D. S. Bradshaw and D. L. Andrews, "Resonance energy transfer: The unified theory revisited", J. Chem. Phys., 119, pp. 2264-2274, 2003.

26. K. El-Hami and K. Matsushige, "Electrostriction in single-walled carbon nanotubes", Ultramicroscopy, 105, pp. 143-147, 2005.

27. R. D. Jenkins, G. J. Daniels and D. L. Andrews, "Quantum pathways for resonance energy transfer", J. Chem. Phys., 120, pp. 11442-11448, 2004.

28. A. D. Buckingham, "Birefringence Resulting from the Application of an Intense Beam of Light to an Isotropic Medium", Proceedings of the Physical Society of London Section B, 69, pp. 344-349, 1956.

29. R. Y. Chiao, E. Garmire and C. H. Townes, "Self-Trapping of Optical Beams", Phys. Rev. Lett., 13, pp. 479$482,1964$. 